\title{
Kinetics and Mechanism of Reduction of Ferric Oxide by Hydrogen
}

\author{
By R. P. Viswanath*, B. Viswanathan* \\ and M. V. C. Sastri*
}

\begin{abstract}
Kinetic studies on the reduction of $\alpha-\mathrm{Fe}_{2} \mathrm{O}_{3}$ with hydrogen have been carried out in the temperature range 300 to $500^{\circ} \mathrm{C}$ through direct weight-loss measurements and differential thermography. The results show that the reduction proceeds by a consecutive two-step mechanism via $\mathrm{Fe}_{3} \mathrm{O}_{4}$, with the overall rate governed by the topochemical reduction of $\mathrm{Fe}_{3} \mathrm{O}_{4}$, to $\mathrm{Fe}$, i.e., the formation and nucleation of metallic iron, rather than by any of the mass transport processes. The phasecomposition of the solid mass have been established by X-ray diffraction, Mössbauer spectroscopy and DTA.
\end{abstract}

(Received April 23, 1976)

\section{Introduction}

The direct reduction of iron oxide with gaseous reducing agents, like hydrogen and carbon monoxide, has attracted considerable attention for scientific investigation in recent years, both on account of its commercial importance and in the context of fundamental considerations of kinetics and mechanism. For the type of gas-solid reaction implied in these processes, various mechanisms have been proposed ${ }^{(1) \sim(7)}$, involving different ratedetermining steps (r.d.s.), such as the following:

(1) the diffusion of gases through the stagnant gas film,

(2) the inward diffusion of hydrogen and/or the outward diffusion of water vapour through the solid product layer,

(3) the topochemical reaction at the gassolid interface,

(4) a mixed control, involving initial surface reaction control changing over subsequently to one of gas-diffusion through the solid product.

This controversial situation calls for detailed kinetic studies to be carried out under welldefined conditions in order to obtain reliable data for a critical evaluation of the claims of

* Department of Chemistry, Indian Institute of Technology, Madras. 600 036, India. mass transport and topochemical processes for the r.d.s. The present paper reports a study of the kinetics of reduction of $\alpha-\mathrm{Fe}_{2} \mathrm{O}_{3}$ with hydrogen, undertaken with this aim.

\section{Experimental}

\section{Materials}

Pure $\alpha-\mathrm{Fe}_{2} \mathrm{O}_{3}$ was prepared by heating pure ferric nitrate (Baker Analysed) at $475^{\circ} \mathrm{C}$ to constant weight $(8 \mathrm{hr})$. Chemical and X-ray analyses confirmed its purity and phaseidentity as $\alpha-\mathrm{Fe}_{2} \mathrm{O}_{3}$. Cylindrical pellets of the oxide ( $5 \mathrm{~mm}$ dia. $\times 5 \mathrm{~mm}$ height) were prepared by compression in stainless steel die at $50 \mathrm{~atm}$. pressure.

Pure dry hydrogen was obtained directly from an 'Elhygen' electrolytic hydrogen generator (Milton Roy Co., St. Petersburg, Fla., U.S.A.) provided with a $\mathrm{Pd}-\mathrm{Ag}$ alloy diffuser tube cathode.

\section{Kinetics measurements}

The rate of reduction was deduced from weight change in a single pellet of oxide (ca. $200 \mathrm{mg}$ ), found with a very sensitive quartzspring balance (sensitivity $=2 \mathrm{mg} / \mathrm{mm}$ ). The latter was mounted inside a Pyrex glass tube (25 mm dia) with gas inlet-outlet connections and heating arrangement. The oxide-pellet (in quartz-bucket) was heated slowly in flowing nitrogen to desired temperature, after stabiliz-

1977 Vol. 18 
ing which, the gas-flow was switched over to hydrogen at the desired rate, and the weightchange was followed as a function of time using a high magnification reading microscope (readability $=0.01 \mathrm{~mm}$ ).

\section{Differential thermographs (DTG)}

Differential thermographs of the reduction were obtained with a Netzsch automatic DTA apparatus (Netzsch Gerätebau $\mathrm{GmbH}$, Selb, Germany-FDR) in flowing hydrogen atmosphere using ignited alumina as reference material.

Isothermal DTG were obtained with the same apparatus, operated at constant temperature $\left(320,340\right.$ and $\left.360^{\circ} \mathrm{C}\right)$. The sample was heated in nitrogen-flow till constancy at the desired temperature was attained and then switched over to hydrogen-flow for reduction.

\section{X-ray diffraction}

X-ray diffractograms of the unreduced, partially reduced and fully reduced samples were recorded with a Philips X-ray diffractometer at a scan speed of $1^{\circ}(2 \theta)$ per minute using $\mathrm{Co}-\mathrm{K}_{\alpha}$ radiation with iron filter.

\section{Mössbauer spectroscopy}

Mössbauer spectra of the samples at various stages of reduction were obtained with a Frieseke and Höpfner apparatus (F and $\mathbf{H}$ $\mathrm{GmbH}$, Erlangen Brück, Germany-FDR) fitted with a Co-57 source in the stainless steel capsule and a $\mathrm{F}$ and $\mathrm{H}$ 400-channel analyser.

\section{Results}

\section{Kinetic data}

Typical isothermal rate-curves for the reduction of $\alpha-\mathrm{Fe}_{2} \mathrm{O}_{3}$ at various flow-rates (80$240 \mathrm{~m} \ell$ per $\mathrm{min}$ ) of hydrogen and at different temperatures are shown in Fig. 1. Since beyond $280 \mathrm{~m} \ell \mathrm{H}_{2}$ per min the rate of reduction was insensitive to flow-rate, this flow-rate of hydrogen was maintained in all subsequent experiments to eliminate effect of flow-rate on the kinetics. Figure 2 shows the degree of reduction (relative per cent weight-loss) as a function of time, at various temperatures in the range 300 to $500^{\circ} \mathrm{C}$ and at $280 \mathrm{~m} \ell / \mathrm{min}$ hydrogen-flow. It is seen that in each of the rate-curve obtained at temperatures below $400^{\circ} \mathrm{C} \&$ hydrogen-flow rates $<280 \mathrm{~m} \ell / \mathrm{min}$, there is a break at a relative per cent weightloss of about 11 (which corresponds to quantitative conversion of $\mathrm{Fe}_{2} \mathrm{O}_{3}$ to $\mathrm{Fe}_{3} \mathrm{O}_{4}$ ) while no such discontinuity is found in the curves for higher temperatures and hydrogen-flowrates. Typical plots relating the rate of reduction with degree of reduction are shown in Fig. 3 from which it can be seen that at the lower temperatures and lower hydrogen-flowrates, the rate peaks up to a sharp maximum at the 11 per cent stage of reduction and then falls to a steady value for the rest of the reduction. A similar observation was reported by Kosyagin and Gasanova ${ }^{(8)}$.

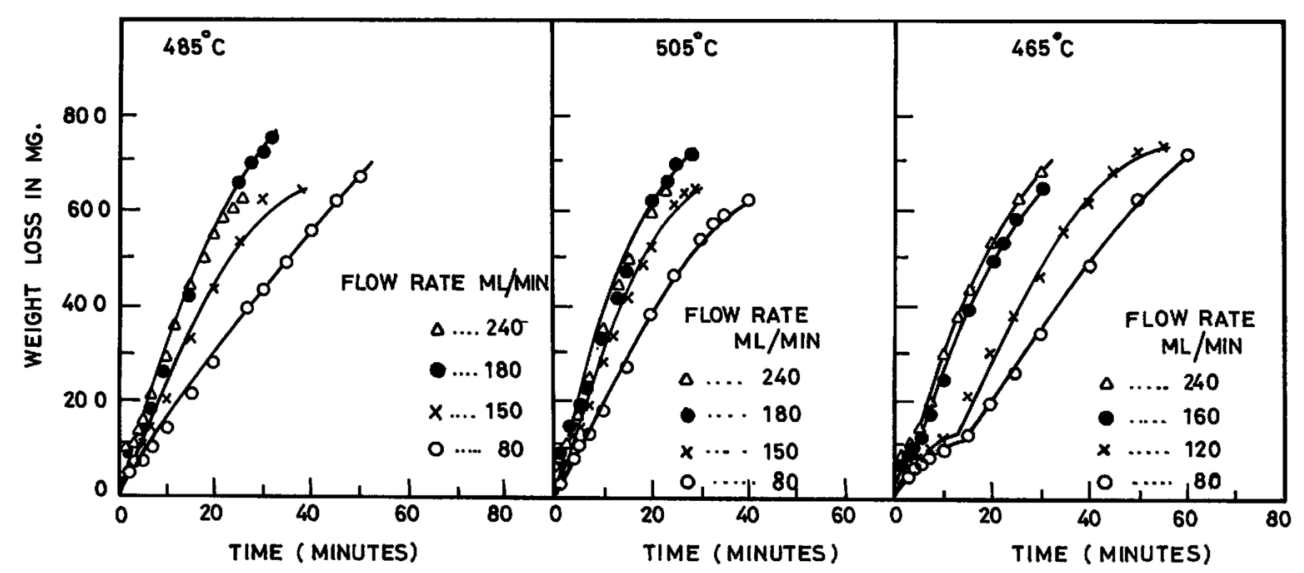

Fig. 1 Primary kinetic curves for the reduction of $\mathrm{Fe}_{2} \mathrm{O}_{3}$ at different flow rates of hydrogen. 

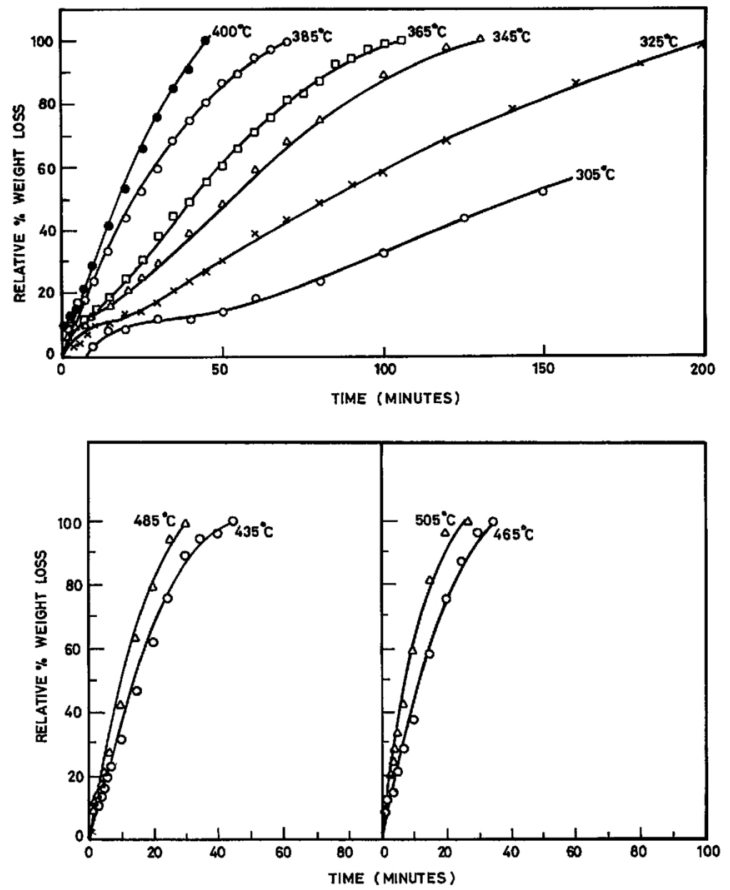

Fig. 2 Effect of temperature on the reduction of $\mathrm{Fe}_{2} \mathrm{O}_{3}$ at constant flow of hydrogen $(280 \mathrm{~m} \ell / \mathrm{min})$.
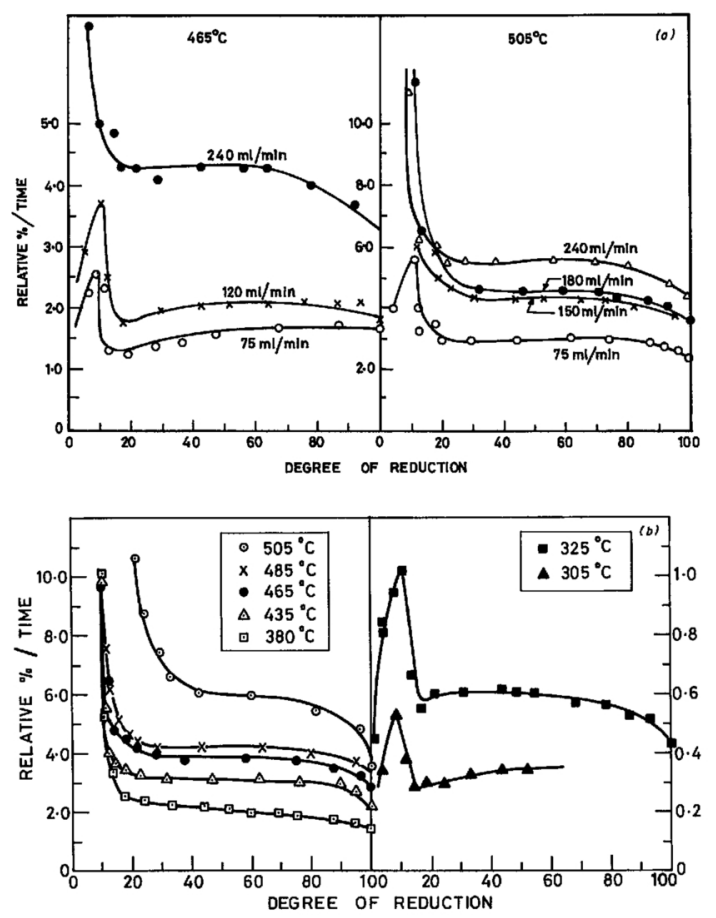

Fig. 3 Effect of the degree of reduction on the rate.

(a) at different flow rates of hydrogen

(b) at different temperatures

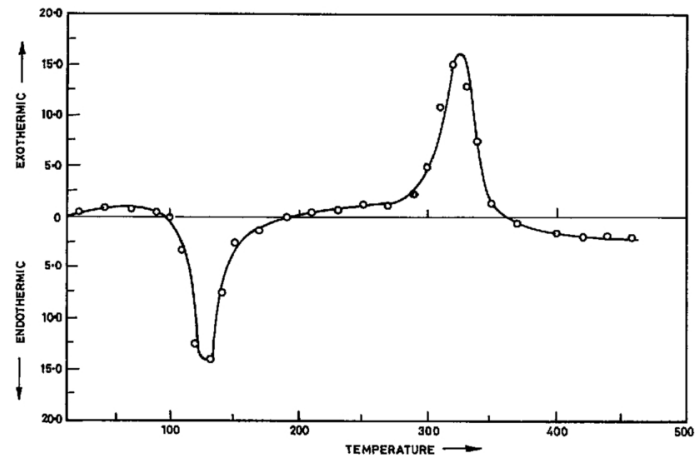

Fig. 4 DTA plot for the reduction of $\mathrm{Fe}_{2} \mathrm{O}_{3}$ in flowing hydrogen.

The normal differential thermographs of the reduction of $\mathrm{Fe}_{2} \mathrm{O}_{3}$ show an exothermic peak around $300-350^{\circ} \mathrm{C}$ followed by a diffuse endothermic peak (Fig. 4). The heat changes occurring at various stages of reduction are manifest in the isothermal DTG, which exhibit a sharp exothermic peak immediately on admission of hydrogen, followed by a slow drift towards the endothermic side. The height of the exothermic peak decreases with increasing temperature.

\section{X-ray data}

The $d$-spacings and intensities deduced from the X-ray diffractograms for $\alpha-\mathrm{Fe}_{2} \mathrm{O}_{3}$ (unreduced, less than 11 per cent reduced and $>11$ per cent reduced) and $\mathrm{Fe}_{3} \mathrm{O}_{4}$ are given in Table 1 . These data reveal that at any stage of reduction no more than two solid phases (either $\mathrm{Fe}_{2} \mathrm{O}_{3}$ and $\mathrm{Fe}_{3} \mathrm{O}_{4}$ at $<11$ per cent reduction or $\mathrm{Fe}_{3} \mathrm{O}_{4}$ and $\mathrm{Fe}$ at $>11$ per cent reduction) are present together. Mössbauer spectra confirm the above conclusion regarding phase-composition of the product (Fig. 5).

\section{Discussion}

The rate-curves obtained at low temperatures and low flow rates (Figs. 2 and 3) suggest a two-step consecutive process for the reduction of ferric oxide, as proposed earlier by Russian and Japanese authors ${ }^{(9)(10)}$, thus:

$$
\begin{aligned}
& 3 \mathrm{Fe}_{2} \mathrm{O}_{3}+\mathrm{H}_{2} \stackrel{k_{1}}{\longrightarrow} 2 \mathrm{Fe}_{3} \mathrm{O}_{4}+\mathrm{H}_{2} \mathrm{O}, \\
& \mathrm{Fe}_{3} \mathrm{O}_{4}+4 \mathrm{H}_{2} \stackrel{k_{2}}{\longrightarrow} 3 \mathrm{Fe}+4 \mathrm{H}_{2} \mathrm{O} .
\end{aligned}
$$


Table 1 X-ray diffraction data for partially reduced ferric oxide samples.

\begin{tabular}{|c|c|c|c|c|c|c|c|c|c|}
\hline \multicolumn{2}{|c|}{$\alpha-\mathrm{Fe}_{2} \mathrm{O}_{3}^{+}$} & \multicolumn{2}{|c|}{$\mathrm{Fe}_{3} \mathrm{O}_{4}^{++}$} & \multicolumn{2}{|c|}{$\alpha-\mathrm{Fe}^{+++}$} & \multicolumn{2}{|c|}{$\begin{array}{c}\mathrm{Fe}_{2} \mathrm{O}_{3} \text { reduced } \\
\text { to less than } \\
11 \text { per cent }\end{array}$} & \multicolumn{2}{|c|}{$\begin{array}{c}\mathrm{Fe}_{2} \mathrm{O}_{3} \text { reduced } \\
\text { to greater than } \\
11 \text { per cent }\end{array}$} \\
\hline$d(\AA)$ & $I / I_{0}$ & $d(\AA)$ & $I / I_{0}$ & $d(\AA)$ & $I / I_{0}$ & $d(\AA)$ & $I / I_{0}$ & $d(\AA \AA)$ & $I / I_{0}$ \\
\hline & & 2.97 & 70.0 & & & 2.97 & 21.7 & 2.97 & 15.7 \\
\hline 2.69 & 100.0 & & & & & 2.69 & 33.0 & & \\
\hline 2.51 & 50.0 & 2.53 & 100.0 & & & 2.53 & 100.0 & 2.53 & 100.0 \\
\hline \multirow[t]{3}{*}{2.20} & 30.0 & & & & & 2.20 & 10.5 & & \\
\hline & & 2.10 & 69.0 & & & 2.10 & 21.7 & 2.10 & 24.5 \\
\hline & & & & 2.025 & 100.0 & & & 2.025 & 33.0 \\
\hline \multirow[t]{2}{*}{1.84} & 40.0 & & & & & 1.84 & 10.6 & & \\
\hline & & 1.71 & 60.0 & & & 1.71 & 7.0 & & \\
\hline 1.69 & 60.0 & & & & & 1.69 & 15.0 & & \\
\hline 1.59 & 16.0 & 1.62 & 85.0 & & & 1.62 & 22.0 & 1.62 & 18.0 \\
\hline 1.49 & 35.0 & 1.49 & 85.0 & 1.47 & 19.0 & 1.49 & 43.3 & 1.49 & 24.0 \\
\hline 1.45 & 32.0 & & & & & 1.45 & 1.0 & & \\
\hline \multirow[t]{2}{*}{1.31} & 20.0 & 1.33 & 20.0 & & & & & & \\
\hline & & 1.28 & 30.0 & & & & & 1.28 & 7.0 \\
\hline 1.16 & 10.0 & 1.26 & 10.0 & 1.17 & 30.0 & & & 1.17 & 14.0 \\
\hline 1.14 & 12.0 & 1.21 & 20.0 & & & & & & \\
\hline 1.05 & 18.0 & 1.12 & 30.0 & 0.906 & 12.0 & & & & \\
\hline
\end{tabular}

Powder diffraction file (Inorganic), Pub., Joint Committee on Powder Diffraction Standards, Pennsylvania, 19103, U.S.A.

File No.: ${ }^{+} 13.0534,++19.0629,+++6.096$.

The observed discontinuities at 11 per cent reduction mark the completion of the first step. At higher temperatures and flow rates, the first step occurs very rapidly and is completed before any reading can be taken, and so, the rate curves show a continuous decline with progress of reduction. Hence, it follows that of the two topochemical processes, the second step $\left(\mathrm{Fe}_{3} \mathrm{O}_{4} \rightarrow \mathrm{Fe}\right)$ is the slower one.

The consecutive two-step mechanism is also supported by the differential thermographs, the sharp exothermic peak corresponding to the rapid first step and broad endothermic band to the slower reduction of $\mathrm{Fe}_{3} \mathrm{O}_{4}$ to $\mathrm{Fe}$. A more detailed study of the kinetics of this process through measurement of heat changes during reduction will be published elsewhere.

With $k_{1} \gg k_{2}$, it is obvious that no more than two of the solid phases can coexist, as is evident from the $\mathrm{X}$-ray and Mössbauer data.

Next, to determine whether it is the topochemical process or any of the mass transport steps that prevails as the overall rate-determining step of the reduction of ferric oxide, the kinetic data have been fitted into various equations applicable to gas-solid reactions ${ }^{(11)}$, with results as follows:

The absence of a linear relationship between the degree of reduction $(x)$ and time $t$ (vide Fig. 2) rules out the possibility of diffusion through stagnant gas film being rate-determining, since the latter requires a fit with the equation $t=k x$. It is definitely ruled out as r.d.s. at hydrogen-flow-rates $\geqslant 280 \mathrm{~m} \ell \mathrm{min}^{-1}$, when the rate becomes independent of the flow-rate.

If gas-diffusion through the solid product layer were rate-determining, the kinetic data should obey the equation

$$
t=k\left[\frac{1}{2}-\frac{x}{3}-\frac{(1-x)^{2 / 3}}{2}\right]
$$

It is seen from the few typical examples presented in Fig. 6 that the kinetic data do not fit in with this equation either.

The rate equation for a gas-solid reaction governed by the topochemical reaction at the interface is ${ }^{(6)(7)}$

$$
t=k\left[1-(1-x)^{1 / 3}\right]
$$



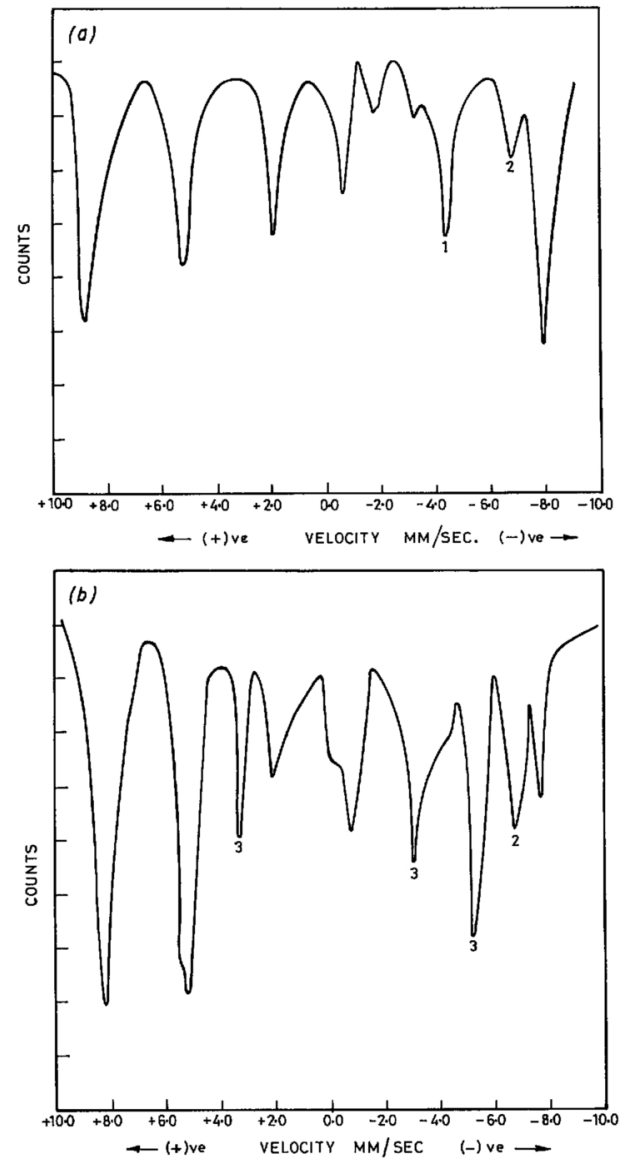

Fig. 5 Mössbauer spectra of $\mathrm{Fe}_{2} \mathrm{O}_{3}$ samples:

(a) reduced to less than 11 per cent reduction, and

(b) reduced to greater than 11 per cent reduction. Phases identified by characteristic lines (1) $\alpha-\mathrm{Fe}_{2} \mathrm{O}_{3}$

(2) $\mathrm{Fe}_{3} \mathrm{O}_{4}$ and (3) metallic $\mathrm{Fe}$.

This can be checked by a linear plot of $t$ vs $\left[1-(1-x)^{1 / 3}\right]$. Since the reduction in the present investigation has been found to be a stepwise process, with $\mathrm{Fe}_{3} \mathrm{O}_{4} \rightarrow \mathrm{Fe}$ being the slow one, the fraction reduced has been corrected to the fraction reduced with respect to the magnetite formed and this is represented as ' $x$ ' and plotted as $\left[1-\left(1-x^{\prime}\right)^{1 / 3}\right]$ vs $t$. That the experimental data fit in very well with this mechanism is substantiated by a few typical plots of equation (4) shown in Fig. 7. The $k$ values evaluated therefrom yield a value of $13.6 \mathrm{kcal} \mathrm{mol} \mathrm{m}^{-1}$ for the apparent activation energy. This agrees reasonably well with the values ranging from 14.9 to $16.5 \mathrm{kcal} \mathrm{mol}^{-1}$ re-

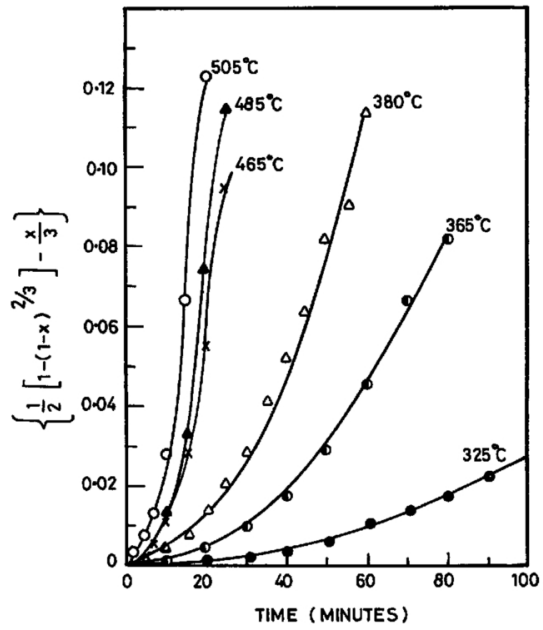

Fig. 6 The kinetics plots, for the model based on gas-diffusion through the solid product layer, for the reduction of $\mathrm{Fe}_{2} \mathrm{O}_{3}$ by hydrogen at constant flow of $280 \mathrm{~m} \ell / \mathrm{min}$.

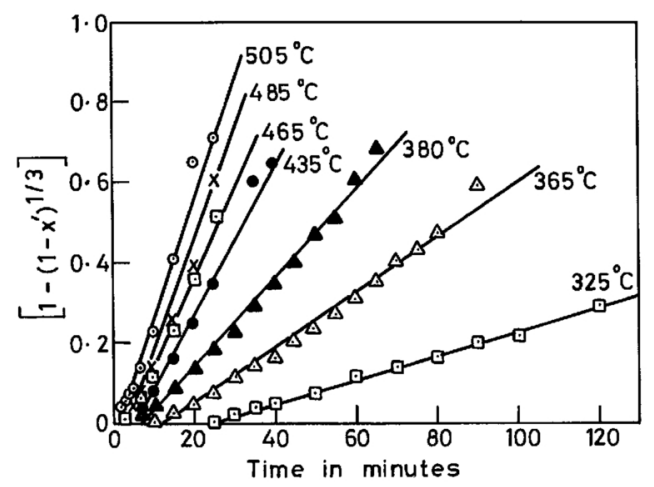

Fig. 7 The kinetics plots, for the topochemical reaction as rate controlling, for the reduction of $\mathrm{Fe}_{3} \mathrm{O}_{4}$ by hydrogen at a constant flow of $280 \mathrm{~m} \ell /$ min.

ported in literature ${ }^{(6)(7)(12)}$.

In the mechanism proposed by Richardson et al. ${ }^{(13)(14)}$ the topochemical reaction involves mainly the diffusion of $\mathrm{Fe}^{2+}$ ions inwards from the reaction-interface, leading eventually to a supersaturation of $\mathrm{Fe}^{2+}$ ions in the bulk of the solid, as the reduction of $\mathrm{Fe}^{3+}$ ions in $\mathrm{Fe}_{3} \mathrm{O}_{4}$ proceeds. Coalescence and further reduction of the supersaturated $\mathrm{Fe}^{2+}$ ions leads to formation of metal nuclei and the growth of the latter is an important step in the overall reaction mechanism. Such a mechanism will account for the applicability of 'contracting 
cube law' (eq. (4)) to the kinetic data reported in the present paper. If, as required by this law, the reduction proceeds by the growth of the metal nuclei in all the three directions, it is implied that the product layer offers little resistance to the diffusion of gases into and away from the reaction-interface.

\section{Conclusion}

From the forgoing it can be concluded that the reduction of pure $\alpha-\mathrm{Fe}_{2} \mathrm{O}_{3}$ by hydrogen (below $575^{\circ} \mathrm{C}$ ), is occurring by a consecutive two-step mechanism, which is controlled by a topochemical reaction at the $\mathrm{Fe}_{3} \mathrm{O}_{4} / \mathrm{Fe}$ interface.

\section{REFERENCES}

(1) S. E. Woods: Disc. Faraday Soc., 4 (1948), 184.

(2) L. V. Bogdandy and W. Janke: Z. Electrochem., 61 (1957), 1146.

(3) J. Henderson: J. Austr. Inst. Metals, 7 (1962), 115.
(4) N. J. Themelis and W. H. Gauvin: Trans. Met. Soc. AIME, 227 (1963), 290.

(5) N. J. Themelis and W. H. Gauvin: A.I.Ch.E.J., 8 (1962), 437.

(6) W. M. McKewan: Trans. Met. Soc. AIME, 212 (1958), 791; Trans. Met. Soc. AIME, 218 (1960), 2.

(7) W. M. McKewan: Trans. Met. Soc. AIME, 224 (1962), 387.

(8) V. G. Kosyagin and L. M. Gasanova: CA 68: $5134 \mathrm{k}$.

(9) L. N. Rudenko and S. T. Rostovtsev: Izv. Vysshik, Uchebn. Zavedeni Chernaya, Met., 5 (11) (1962), 5 (CA.58: 7650f)).

(10) M. Yano, J. Imoto, and A. Moriyama: Kogyo Kagaku Zasshi, 65 (1962), 485 (CA.57: 9514h).

(11) O. Levenspiel: Chemical Reaction Engineering, John Wiley and Sons, Inc., New York-London, (1966), p. 338.

(12) P. C. Ghosh: Trans. Indian Inst. Metals, 16 (1963), 35.

(13) F. D. Richardson and E. Dancy: Disc. Faraday Soc., 4 (1948), 229.

(14) O. H. Gellner and F. D. Richardson: Nature, 168 (1951) 23. 